\title{
OBESITY AND DYSLIPIDEMIA IN CHILDREN WITH CHRONIC HEPATITIS
}

\author{
Alice N. Azoicai, Bogdan A. Stana, Paula Popovici, Ileana K. Ioniuc, \\ Monica M. Alexoae, Irina Criscov, Alina M. Murgu, Evelina Moraru \\ $2^{\text {nd }}$ Clinic of Pediatrics, "Sf. Maria” Emergency Hospital for Children, \\ "Gr. T. Popa" University of Medicine and Pharmacy, Iasi, Romania
}

\begin{abstract}
Chronic hepatitis association with various types of dyslipidemia is a reality not only for the obese pediatric patient, in which we expect to find hypercholesterolemia and / or hypertriglyceridemias, but also for normal weight children with hepatitis. The causes of this association are multiple, but we need to consider first the innate predisposition and transmission of genetic forms of dyslipidemia. Meanwhile, some secondary dyslipidemia are chronic liver disease related. Those considerations are forming a vicious circle in which primary treatment of metabolic imbalances has a great importance for the success of antiviral therapy.
\end{abstract}

Keywords: hepatitis, dyslipidemia, obesity, children

\section{BACKGROUND}

The prevalence of dyslipidemia in patients with chronic hepatitis undergoing antiviral therapy can be attributed to the effect of Interferon which, like antiretroviral therapy, causes a reduction of CD4 positive lymphocytes, a decreasing BMI, modifying individual characteristics of metabolic individuals and changing new or preexisting pathological conditions. $(1,2)$ Most studies recognize that protease inhibitors are risk factors in development of dyslipidemia, besides other mechanisms such as inhibition of lipoprotein lipase, induction of insulin resistance versus lipolysis or lipogenesis homeostasis disorder caused by viral infection itself. (3) Also, there is evidence supporting the incentive effect of lipodystrophy and the redistribution of body fat that reverse transcriptase inhibitors have in non-nucleoside naive patients who receive treatment with protease inhibitors (3-5). Given that reasons and the balance of chronic hepatitis antiviral therapy, these side effects should be carefully monitored, because they may cause serious drop in compliance to therapy, even discontinuation or reconsideration of treatment options. $(6,7)$

\section{MATERIAL AND METHODS}

The study cohort was formed by 166 patients (children and adolescents) diagnosed with viral chronic hepatitis, in evidences of $2^{\text {nd }}$ Pediatric Clinic of „Sf. Maria” Emergency Hospital for Children, in Iasi, Romania. Authors reviewed BMI status, serum cholesterol and triglycerides, and the treatment status (antiviral or hepatoprotective therapy). Furthermore, we evidenced several correlations between overweight/obesity and dyslipidemia (hypercholesterolemia, hypertriglyceridemia, or mixed dyslipidemia).

\section{RESULTS}

Regarding the presence of obesity in patients with chronic hepatitis, authors highlighted semificative prevalence of dyslipidemia (68.7\%), with high value of statistical parameters $\chi^{2}=9.25$, $\mathrm{p}=0.0023$ ).

Correlational analysis confirms a highly significant relation between obesity and dyslipidemia, slipidemiilor $(r=0.71, p=0.0009,95 \% \mathrm{CI})$. These results underline a clear importance in studying dysmetabolic status as a risk factor and as a marker for prognostic evaluation. Furthermore, the therapeutic response can be seriously influenced by the presence of obesity and serum lipid disorders.

Evaluating the odd ratio in developing dyslipidemia, the authors demonstrated a retrospective risk of 5.66 times higher in obese patients. Regarding

Corresponding author:

Bogdan A. Stana, “Gr. T. Popa” University of Medicine and Pharmacy, 16 University Street, lasi, Romania

E-mail: bogdan.stana@gmail.com 


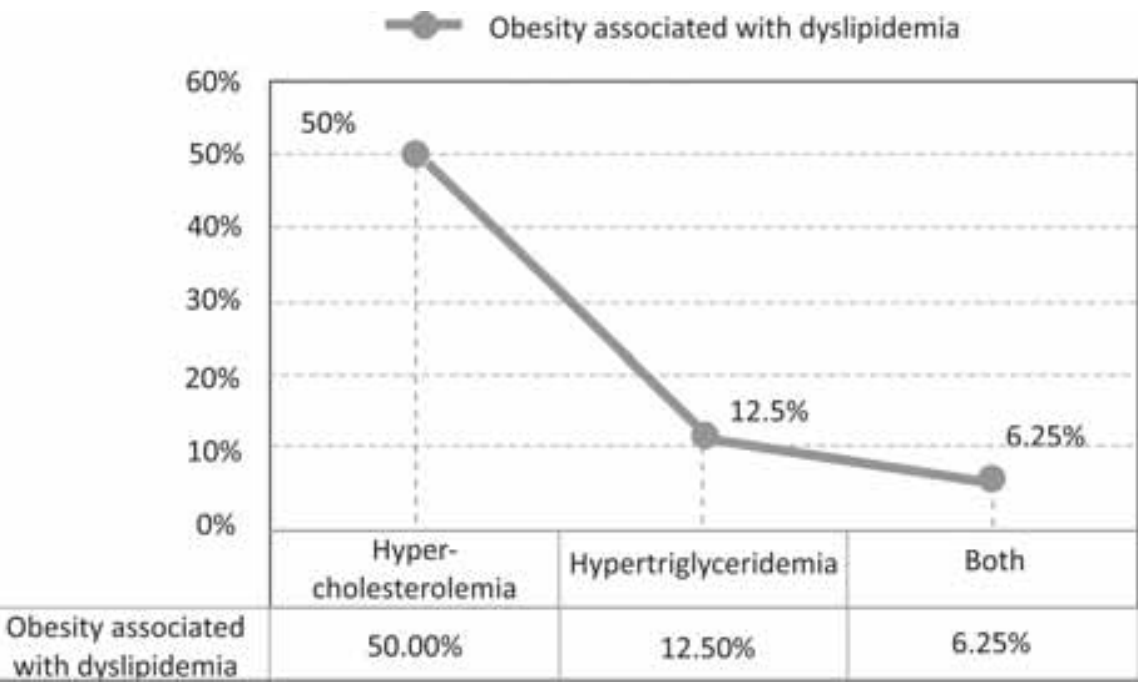

FIGURE 1. Association between obesity and dyslipidemia

TABLE 1. Statistical parameters regarding association between dyslipidemia and obesity

\begin{tabular}{|l|c|c|}
\hline & $\chi^{2}$ & $\begin{array}{c}\mathbf{p} \\
\text { 95\% confidence interval }\end{array}$ \\
\hline Pearson $-\chi^{\mathbf{2}}$ & 11.05 & 0.0008886 \\
\hline Yates & 9.25 & 0.0023537 \\
\hline $\begin{array}{l}\text { Correlation coefficient } \\
\text { (Spearman Rank R) }\end{array}$ & 0.713 & 0.0009211 \\
\hline
\end{tabular}

TABLE 2. Odd ratio and risk ratio in dyslipidemia

\begin{tabular}{|c|c|c|c|}
\hline & \multirow{2}{*}{$\begin{array}{l}\text { Estimated } \\
\text { value }\end{array}$} & \multicolumn{2}{|c|}{ 95\% confidence interval } \\
\hline & & Minimum & Maximum \\
\hline \multicolumn{4}{|c|}{ Chance parameters } \\
\hline (Odd ratio) & 5.66 & 1.68 & 20.06 \\
\hline \multicolumn{4}{|c|}{ Risk parameters } \\
\hline (Risk ratio) & 2.46 & 1.62 & 3.73 \\
\hline
\end{tabular}

the results of study cohort we can estimate a prospective risk of 2.46 , with a risk ratio of 2.46 , as calculated above.
Relative frequency of obese patient with chronic hepatitis increased in the first 60 months from the baseline (the moment of study inclusion of the patient), from $4.18 \%$ (the moment of establishing the chronic hepatitis diagnostic) to $15.38 \%$ (after 48 months from the moment of initiating antiviral or hepatoprotective therapy).

Parametric results demonstrate a significant association between the type of treatment and obesity, regarding BMI dynamic fluctuation. This statistic aspect was highlighted by the high value of correlation coefficient $(\mathrm{r}=0.642, \mathrm{p}=0.0340,95 \% \mathrm{CI})$, and also by the corrected M-L (M-L $\chi^{2}=18.9$, $\mathrm{p}=0.025,95 \% \mathrm{CI})$. Relative ratio was obtained fractioning the total number of cases that could be evaluated in that particular moment.

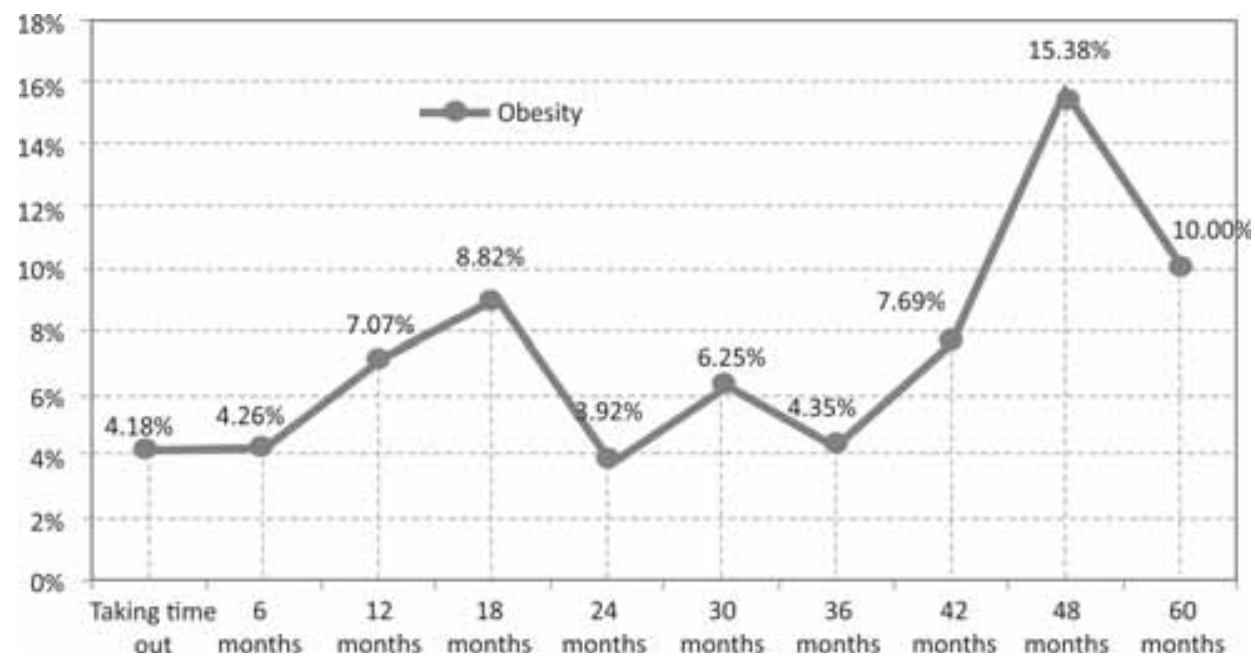

FIGURE 2. Dynamic frequency of obesity (baseline) 


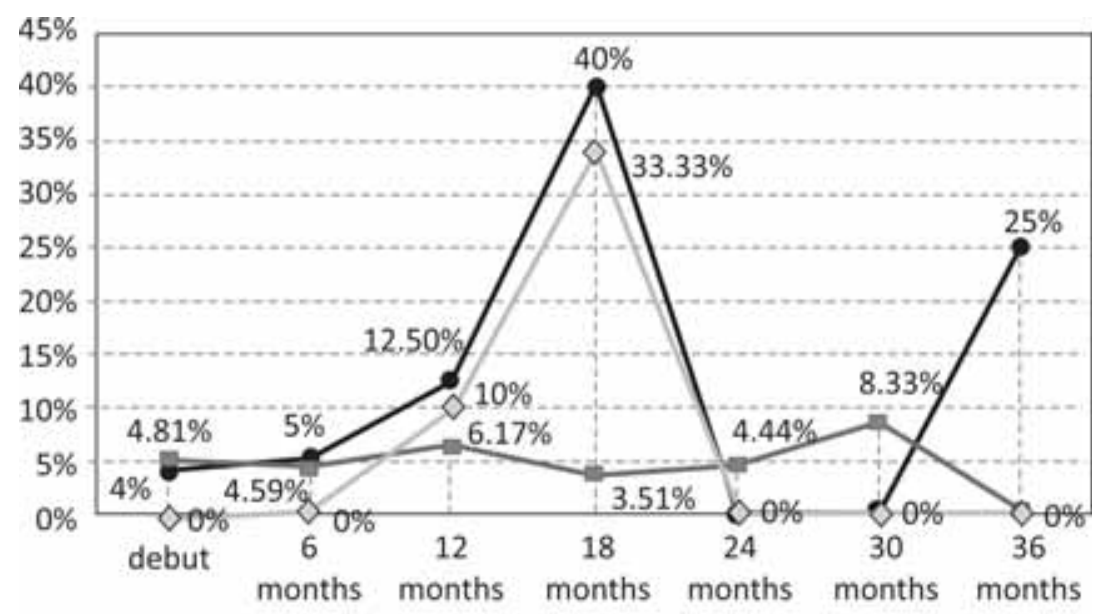

FIGURE 3. Dynamic frequency of obesity (therapy initiation)

TABLE 4. Parameters for associating antiviral therapy and obesity

\begin{tabular}{|l|c|c|}
\hline$(\mathrm{df}=9)$ & $\chi^{\mathbf{2}}$ & $\begin{array}{c}\mathbf{p} \\
\text { 95\% confidence interval }\end{array}$ \\
\hline$\chi^{\mathbf{2}}$ & 18.98101 & 0.02536 \\
\hline M-L corrected & 13.95425 & 0.012398 \\
\hline $\begin{array}{l}\text { Correlation coefficient } \\
\text { (Spearman Rank R) }\end{array}$ & 0.6423218 & 0.034079 \\
\hline
\end{tabular}

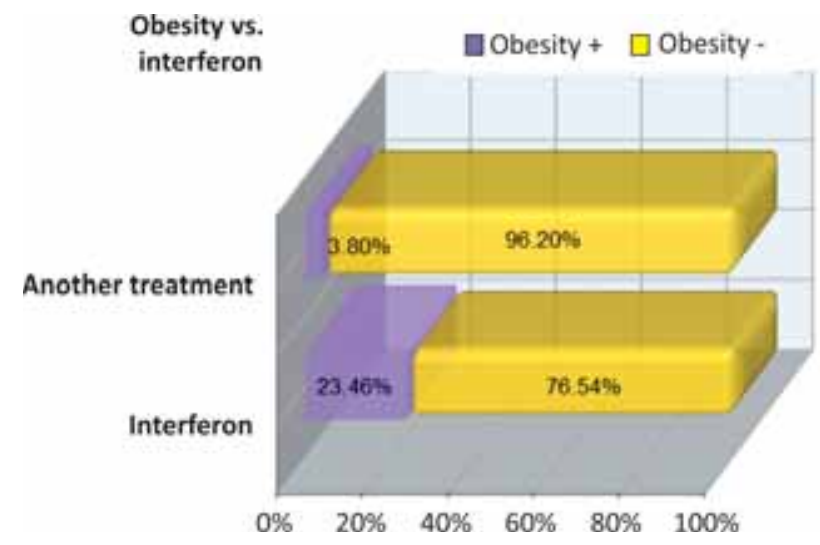

FIGURE 4. Obesity and Interferon therapy

In cases treated with specific antiviral therapyInterferon, the authors highlighted a significant increase of obese patients $(23.46 \%)$, comparative with children receiving non-specific therapy; those findings were underlined also by the high value of statistical parameters $\left(\chi^{2}=20.05, \mathrm{p}=0.0000076\right)$.

Although the literature postulates the reverse results in Interferon therapy (most patients lose weight during treatment) $(8,9)$ the increased BMI can be interpreted with hypercaloric and hyperproteic lifestyle after completing a therapy cycle. Furthermore, pre-existing obesity (before initiating the antiviral therapy) and even weight loss during therapy (as a risk factor for overweight) could explain those controversies.(10)

\section{CONCLUSIONS}

Study results suggest the need for a real investigation of dyslipidemia in patients with chronic hepatitis initially, but also during therapy, and furthermore-to adapt the treatment. The actual importance of chronic hepatitis diagnosis in developing dyslipidemia and obesity in uncertain, given the coexistence of phenotypic traits, genotypic and/or dietary particularities.

Our study noted a favorable trend in obesity, with a lower overweight rate and body mass index in patients who received antiviral treatment with interferon alpha; in some cases weight loss correlates with decreasing severity of hepatic steatosis.

Statins and fibrates should be taken with reluctance because of the muscle toxic effects, knowing that chronic hepatitis infection is commonly associated with extrahepatic manifestations like myalgia, myositis or rhabdomyolysis. It is therefore appropriate to establish a balance between risk and benefit in case of special categories of patients, especially in cases of coronary heart disease due to dyslipidemia.

As there are no guidelines for treating dyslipidemia in pediatric patients with chronic hepatitis, we must comply with international recommendations regarding the management of hypertriglyceridemia and hypercholesterolemia (diet and medication), and conducting studies to allow the formulation of clear direction in treating these patients. 


\section{REFERENCES}

1. Negro F., Clement S. Impact of obesity, steatosis and insulin resistance on progression and response to therapy of hepatitis $C, J$ of Vir Hep, Oct 2009, 16(10):681-688

2. Su T.C. Chronic hepatitis B virus infection and dyslipidemia, Journal of the Formosan Medical Association, 2004, vol. 103, n4, pp. 286-291

3. Pyrsopoulos N. Extrahepatic manifestations of chronic viral hepatitis, Current Gastroenterol Rep, 3(1), 71-78

4. C.J. Liu, J.H. Kao, D.S. Chen. Therapeutic implications of hepatitis B virus genotypes. Liver Int 2005; 25: 1097-107

5. Chan H.L., Heathcote, Marcellin P.E.J., et al. Treatment of hepatitis B e antigen positive chronic hepatitis with telbivudine or adefovir: a randomized trial. Ann Intern Med 2007; 147: 745-54

6. Baig S., The Extrahepatic Manifestations of Hepatitis B Virus, J Coll Physicians Surg Pak, 2008, 18 (7): 451-457
7. Bressler B.L., Guindi M., Tomlinson G., Heathcote J. High body mass index is an independent risk factor for nonresponse to antiviral treatment in chronic hepatitis C. Hepatology 2003; 38: 639-644.

8. Cammà C., Di Bona D., Schepis F., Heathcote E.J., Zeuzem S., Pockros P.J., et al. Effect of peginterferon alfa-2a on liver histology in chronic hepatitis $\mathrm{C}$ : a meta-analysis of individual patient data. HEPATOLOGY 2004; 39: 333-342.

9. Younossi Z.M., McCullough A.J., Ong J.P., Barnes D.S., Post A., Tavill A., et al. Obesity and non-alcoholic fatty liver disease in chronic hepatitis C. J Clin Gastroenterol 2004; 38: 705-709.

10. Patton H.M., Patel K., Behling C., Bylund D., Blatt L.M., Vallée M., et al. The impact of steatosis on disease progression and early and sustained treatment response in chronic hepatitis $\mathrm{C}$ patients. J Hepatol 2004; 40: 484-490 